Bangladesh Journal of Bioethics 2014; 5(1):5-10

\title{
INSTITUTIONAL REVIEW BOARD (IRB): ITS ROLE AND RESPONSIBILITY IN MAKING RESEARCH ETHICAL
}

\author{
Dr. Abu Sadat Mohammad Nurunnabi \\ Bangladesh Medical Research Council, Dhaka, Bangladesh. \\ Email: shekhor19@yahoo.com
}

ABSTRACT: This is a review article prepared as a part of the assignment in the educational activity and training on research ethics titled "Ethical and Regulatory Aspects of Clinical Research" arranged by the Bangladesh Bioethics Society (BBS), Dhaka, Bangladesh, in collaboration with the Department of Bioethics of National Institutes of Health (NIH), Bethesda, Maryland, USA, through video conferencing between September 25 and November 13 of 2013. The search was confined to 'Google', 'HINARI' and 'PubMed' published articles. Besides, some guidelines on roles and responsibilities of Institutional Review Board (IRB) were taken into consideration. Key words used for searching were 'institutional review board', 'ethical review committee' and 'ethical research'. A total of 18 journal articles and some guidelines were selected for this writing. The systemic review from the databases revealed some important discussions on research, ethical research, roles and responsibilities of IRB/ERC and its challenges, and national/institutional research strategies.

Key words: Institutional review board (IRB), ethical review committee (ERC), ethical research, research strategy.

INTRODUCTION: An Institutional Review Board (IRB), also known as an independent ethics committee or ethical review board, is a committee that has been formally designated to approve, monitor, and review biomedical and behavioral research involving humans ${ }^{1}$. They often conduct some form of risk-benefit analysis in an attempt to determine whether or not research should be done. The number one priority of IRBs is to protect human participants from physical or psychological harm ${ }^{2}$. In the United States, the Food and Drug Administration (FDA) and Department of Health and Human Services (HHS) (specifically Office for Human Research Protections) regulations have empowered IRBs to approve, require modifications in planned research prior to approval, or disapprove research. IRBs are responsible for critical oversight functions for research conducted on human participants that are "scientific," "ethical," and "regulatory" ${ }^{3}$.

Although designed to protect the research participants, IRBs have been criticized by bioethicists for conflicts of interest resulting in lax oversight. As of 2005, the for-profit Western Institutional Review Board claimed to conduct the majority of reviews for new drug submissions to the FDA ${ }^{4}$. In a 2006 study of 575 IRB members at university medical centers, over one-third reported industry financial ties and over one-third admitted they "rarely or never" disclosed conflicts of interest to other board members ${ }^{5}$. A 2009 sting operation by the Government Accountability Office led Coast IRB to shut down after approving the fake product "Adhesiabloc", called "the riskiest thing I've ever seen on this board" by one of the IRBs not approving the fake products ${ }^{6}$.

Originally, IRBs are committees at academic institutions and medical facilities to monitor research studies involving human participants, primarily to minimize or avoid ethical problems ${ }^{7}$. More accurately to say, conflict of interest of the researchers, indemnity for the protection of participants and confidentiality of data are widely accepted as core ethical issues in any research ${ }^{8}$. However, the Belmont Report ${ }^{9}$ exists because of the unfortunate history of unethical 
research conducted on human participants in earlier times. This review has been prepared to highlight some of the key issues on roles and responsibilities of IRB to make a research ethical.

METHODS:This is a review article prepared as a part of the assignment in the educational activity and training on research ethics titled "Ethical and Regulatory Aspects of Clinical Research" arranged by the Bangladesh Bioethics Society (BBS), Dhaka, Bangladesh, in collaboration with the Department of Bioethics of National Institutes of Health (NIH), Bethesda, Maryland, USA, through video conferencing between September 25 and November 13 of 2013. The search was confined to Google, HINARI and PubMed published articles. Besides, some guidelines on roles and responsibilities of IRB were taken into consideration. Key words used for searching were 'institutional review board', 'ethical review committee' and 'ethical research'. A total of 18 journal articles and some guidelines were collected and selected for this writing.

RESULTS: The systemic review from the databases revealed some important discussions on research, ethical research, roles and responsibilities of IRB/ERC and its challenges, and national/institutional research strategies. Research is defined as a systematic investigation designed to develop or contribute to generalized knowledge, or investigation designed to test a hypothesis ${ }^{10}$. The pursuit of new knowledge is considered one of the core missions of almost all institutions. To advance these missions, they must be committed to ensuring that research activities are conducted properly and consistently with the principles of openness, trust, and respect for the human participants. Any systematic investigation (including curricular evaluation) that is designed to develop or contribute to generalized knowledge, and which uses living humans or identifiable private information about living humans, qualifies as human subjects research ${ }^{11}$. Risk refers to the probability of harm or discomfort (these can be physical, psychological, social or economic) occurring as a result of participation in a research study. Both the probability and the magnitude of possible harm may vary from minimal to significant ${ }^{12}$. However, a risk is minimal when the probability and magnitude of harm or discomfort anticipated in the proposed research are not greater than those encountered in daily life ${ }^{13}$. The Department of Health and Human Services [45 CFR 46.111(b)] and Food and Drug Administration [21 CFR 56.111(b)] regulations require that additional safeguards be in place to protect the rights and welfare of research subjects "when all or some of the subjects are likely to be vulnerable to coercion or undue influence". The regulations do not provide a definitive list of vulnerable populations; they leave it largely to the discretion of the IRB. Some populations/groups that may be considered "vulnerable" include: pregnant women, children, prisoners, mentally challenged individuals, economically or educationally disadvantaged individuals and groups, etc. ${ }^{14}$. Research that specifically targets a vulnerable population will receive a higher level of scrutiny than other protocols. This additional scrutiny is to insure that individuals are not being coerced into participation, that the consent process is culturally appropriate, etc. It is also important to remember that vulnerability must be considered in terms of the specific research proposed - not all groups are vulnerable in all situations ${ }^{15}$.

Every research institute should formulate a policy statement, which should be made available to the IRB. It should mention the type of research it will undertake, e.g. pre-clinical toxicology, animal studies in pharmacology or pathology, epidemiological surveys, clinical trials involving patients and studies using healthy volunteers, depending on its resources and infrastructure ${ }^{16}$. This will help in deciding the types of studies to be avoided. The purpose of research i.e. the benefits to be expected from the point of view of its staff, clients, students, society in general or the local community and the institute itself should be revealed in this policy ${ }^{16}$. 
Some IRB reviews are conducted by for-profit organizations known as 'independent' or 'commercial' IRBs. The responsibilities of these IRBs are identical to those based at academic or medical institutions, and they are governed by the same federal regulations. The composition of an IRB for the FDA's requirements ${ }^{17}$ is set in 21 CFR 56.107.

(a) 1 The IRB must have at least five members.

(a) 2 The members must have enough experience, expertise, and diversity to make an informed decision on whether the research is ethical, informed consent is sufficient, and appropriate safeguards have been put in place.

(a) 3 If the IRB works with studies that include vulnerable populations, the IRB should have members who are familiar with these groups. It is common for an IRB to include an advocate for prisoners when considering research that involves them.

(b) 1 The IRB should include both men and women, as long as they aren't chosen specifically for their gender.

(b) 2 The members of the IRB must not be all of the same profession.

(c) The IRB must include at least one scientist and at least one non-scientist. These terms are not defined in the regulations.

(d) The IRB must include at least one person who is not affiliated with the institution or in the immediate family of a person affiliated with the institution. These are commonly called "Community Members."

(e) IRB members may not vote on their own projects.

(f) The IRB may include consultants in their discussions to meet requirements for expertise or diversity, but only actual IRB members may vote.

To vote on a proposal, more than half of the members of the board must be present and there must be a nonscientist present. Exceptions for expedited review are available, where only the chair of the committee or a designee reviews research, but these are relatively narrow ${ }^{17}$.

The primary ethical principles in human subjects review are outlined in the Belmont Report', and include 'respect for persons', 'beneficence', and 'justice'. It clearly explains the three principles that are the main tools that all IRB members should use to evaluate the ethics of specific research proposals. Respect for persons is the first principle which mandates that subjects voluntarily consent to participate in research, that they are adequately and thoroughly informed about the research and what is required, and that their privacy and confidentiality are protected. Beneficence is the second principle which mandates the risks of research are justified by potential benefits to the individual or society and that those risks are minimized. Justice is the third and final principle which mandates the equitable distribution of risks and benefits among those who may benefit from the research, meaning that one subset of a population should not take on all the burden of risk and reap all of the rewards; risks and rewards should be applicable and available to all subsets of a community. The IRB may only approve research for which there is a bona fide informed consent process for participants, for which the risks to subjects are balanced by potential benefits to society, and for which the selection of subjects presents a fair or just distribution of risks and benefits to eligible participants $^{18}$.

The IRB/ERC should review a proposed clinical trial within a reasonable time and document its views in writing, clearly identifying the trial, the documents reviewed and the dates for the following ${ }^{19}$ : 
- approval/favourable opinion

- modifications required prior to its approval/favourable opinion

- disapproval/negative opinion

- termination/suspension of any prior approval/favourable opinion

According to International Conference on Harmonisation - Good Clinical Practice (ICH-GCP) ${ }^{20}$, an IRB should safeguard the rights, safety, and well-being of all trial subjects. the IRB should consider the qualifications of the investigator for the proposed trial, as documented by a current curriculum vitae and/or by any other relevant documentation the IRB requests. Moreover, the IRB/IEC should conduct continuing review of each ongoing trial at intervals appropriate to the degree of risk to human subjects, but at least once per year. The IRB may request more information when in the judgment of the IRB the additional information would add meaningfully to the protection of the rights, safety and/or well-being of the subjects. When a nontherapeutic trial is to be carried out with the consent of the subject's legally acceptable representative, the IRB should determine that the proposed protocol and/or other document(s) adequately addresses relevant ethical concerns and meets applicable regulatory requirements for such trials. Where the protocol indicates prior consent of the trial subject or the subject's legally acceptable representative is not possible, the IRB/ERC should determine that the proposed protocol and/or other document(s) adequately addresses relevant ethical concerns and meets applicable regulatory requirements for such trials (i.e., in emergency situations). The IRB should review both the amount and method of payment to subjects to assure neither presents problems of coercion or undue influence on the trial subjects. Payments to a subject should be prorated and not wholly contingent on completion of the trial by the subject. Last but not the least, the IRB should ensure the information regarding payment to subjects, including the methods, amounts, and schedule of payment to trial subjects, is set forth in the written informed consent form and any other written information to be provided to subjects. The way payment will be prorated should be specified.

The purpose of institutional review boards or research ethics committees or ethical review committees is to ensure that studies involving human research participants are designed to conform the relevant ethical standards and that the rights and welfare of participants are protected ${ }^{21}$. Research ethics committees should not function under political control or one's sweet will ${ }^{22}$. Ethics committees must be independent of research organizations. This independence relates to their decisions, not their operating processes ${ }^{23}$. In spite of the roles and responsibilities of IRBs, the fact is that many are overloaded, understaffed and faced with a variety of skeptical criticism. Many IRBs are lacking the resources and staff to carry out the hefty task of reviewing research ${ }^{24,25}$.

BANGLADESH SCENARIO: It is praise-worthy that Bangabandhu Sheikh Mujib Medical University (BSMMU), the one and only medical university of the country, National Institute of Preventive and Social Medicine (NIPSOM), all the government medical colleges and different specialized government health institutes have got their own institutional review boards (IRBs) or ethical review committees (ERCs) for conducting research by the faculties, physicians, residents and students. However, several challenges have been identified in monitoring function of IRBs on ongoing research in terms of annual review, consent, adherence to protocol, data integrity, auditing and quality assurance ${ }^{2}$. Bangladesh Medical Research Council (BMRC), the focal point for all biomedical research in the country, has got a strong 'National Research Ethics Committee' to review the ethical aspects of a research project ${ }^{26}$. This committee was established in 1979. At present committee consists of 11 members. The clinicians, lawyers, laypersons and 
religious leaders are included as members. The Committee is formed by the Executive Committee of the BMRC and has tenure of three years. The committee is registered in the Office for Human Resource Protection in the USA as an Official Institutional Review Board and it has federal wide assurance ${ }^{2}$.

What is needed in our country is that all institutional review boards (IRBs) in Bangladesh should be operated in a standard fashion, providing an impartial, unbiased review and quick decision and thus facilitating a healthy atmosphere for ethical research in biomedical science in our country ${ }^{8}$. Directorate General of Health Services (DGHS), Bangladesh, published a 'National Health Research Strategy' in 2009 with technical assistance from Bangladesh Medical Research Council (BMRC) and World Health Organization (WHO) featuring different aspects of biomedical research which include institutional framework, priority setting, ethical clearance, financing, monitoring and evaluation, capacity development, dissemination and utilization of research results ${ }^{27}$. Understanding the function of the IRB requires an appreciation for the rules by which it is governed, as well as the history and circumstances that influenced the creation of those rules. Researchers who appreciate the IRB's purpose will be better equipped to navigate the labyrinth of research guidelines and regulations ${ }^{28}$.

CONCLUSION: Biomedical research has expanded tremendously in the past few decades and consequently there has been growing interest in the ethical guidelines that are being followed for the protection of human participants. Toward that end and consistent with existing regulations, institutional review board should formulate policies and procedures to ensure that financial and non-financial relationships with sponsors or other external entities related to the conduct or review of research do not adversely affect the rights and welfare of research volunteers or the integrity of the research.

\section{REFERENCES:}

1. The Office of Human Research Protection. Institutional Review Board Guidebook. Chapter 3, Section A: Risk/Benefit Analysis. p. 1-10. Available from: http://www.saylor.org/site/wpcontent/uploads/2011/08/PSYCH202A-3.1.4-Institutional-Review-Board.pdf. [Retrieved November 30, 2013].

2. Talukder MHK, Hossain MZ, Akhter N, Perveen IA. Institutional Ethical Review Board (IERB): concept \& contect. Bangladesh J Bioethics 2011; 2(2): 24-5.

3. Emanuel EJ, Crouch RA, Arras JD, Moreno JD, Grady C. Ethical and Regulatory aspects of clinical research: readings and commentary. Baltimore: Johns Hopkins University Press; 2003: p.7-20.

4. Elliott C, Lemmens T. Ethics for sale: For-profit ethical review, coming to a clinical trial near you. http://www.slate.com/articles/health and science/medical examiner/2005/12/ethics for sale.html [Retrieved November 30, 2013].

5. Alliance for Human Research Protection. Hospital IRBs are "On the Take"--Tainted by Conflicts of Interest NEJM. Available from: http://www.ahrp.org/cms/content/view/402/100 [Retrieved November 30, 2013].

6. United States Government Accountability Office. Human subjects research: undercover tests show the institutional review board system is vulnerable to unethical manipulation. Available from: http://www.gao.gov/new.items/d09448t.pdf . [Retrieved November 30, 2013].

7. Wolf LE, Croughan M, Lo B. The challenges of IRB review and human subjects protections in practicebased research. Med Care 2002; 40(6): 521-9. 
8. Nurunnabi ASM, Jahan MU, Tanira S. Ethical issues in public health research. Bangladesh J Bioethics 2010; 1(3): 15-21.

9. Rice TW. The historical, ethical, and legal background of human-subjects research. Respir Care 2008; 53(10): 1325-9.

10. Freshwater D, Sherwood G, Drury V. International research collaboration. Issues, benefits and challenges of the global network. J Res Nursing 2006; 11(4): 9295-303.

11. Schwenzer KJ. Protecting vulnerable subjects in clinical research: children, pregnant women, prisoners, and employees. Respir Care 2008; 53(10): 1342-9

12. Orticio LP. Protecting human subjects in research. Insight 2009; 34(3): 14-6.

13. MacDougall DS. Human subject protection in clinical trials. J Int Assoc Physicians AIDS Care 1997; 3(1): 1923.

14. Fleischman AR. Regulating research with human subjects: Is the system broken? Trans Am Clin Climatol Assoc 2005; 116: 91-102.

15. National Institutes of Health. Guidelines for the conduct of research involving human subjects at the National Institutes of Health. 2004. http://ohsr.od.nih.gov/guidelines/GrayBooklet82404.pdf . [Retrieved November 19, 2013].

16. Shrotri DS. Role of ethics committees in medical research. Indian J Med Ethics 2004; 1(4): 121.

17. Code of Federal Regulations. Title 21: Food and Drugs. Chapter I: Food and Drug Administration, Department Of Health And Human Services. Subchapter A: General. Part 56: Institutional Review Boards. Subpart B: Organization and Personnel. http://cfr.vlex.com/vid/56-107-irb-membership-19703309 . [Retrieved November 19, 2013].

18. Pech C, Cob N, Cejka JT. Understanding institutional review boards: practical guidance to the IRB review process. Nutr Clin Pract 2007; 22(6): 618-28.

19. International Conference on Harmonisation of technical requirements for registration of pharmaceuticals for human use. http://ichgcp.net/3-institutional-review-boardindependent-ethics-committee-irbiec [Retrieved November 19, 2013].

20. Skolnick BE. Ethical and institutional review board issues. Adv Neurol 1998; 76: 253-62.

21. Fairchild AL, Bayer R. Ethics and the conduct of public health surveillance. Science 2004; 303: 631-2.

22. Wald SD. Bureaucracy of ethics applications. BMJ 2002; 329: 282-4.

23. Pattison J, Stacey T. Research bureaucracy in the United Kingdom: seeking a balance: response from the Department of Health and COREC. BMJ 2004; 329(7466): 622.

24. Kim WO. Institutional review board (IRB) and ethical issues in clinical research. Korean J Anesthesiol 2012; 62(1): 3-12.

25. Straight TM. Clinical research regulation: challenges to the institutional review board system. Clin Dermatol 2009; 27(4): 375-83.

26. Bangladesh Medical Research Council (BMRC). RICH. Dhaka: 2008.

27. Directorate General of Health Services (DGHS). National Health Research Strategy. Ministry of Health and Family Welfare, Government of the People's Republic of Bangladesh, Dhaka: 2009.

28. Hart R, Belotto M. The institutional review board. Semin Nucl Med 2010; 40(5): 385-92. 